\title{
LAS PRÁCTICAS SOCIALES Y LA REINCIDENCIA DE PERSONAS EN PROCESO DE REINTEGRACIÓN, EN EL MARCO DE LA POLÍTICA NACIONAL DE REINTEGRACIÓN ECONÓMICA Y SOCIAL
}

\author{
PÁMELA CONSTANZA SANTA-MONTOYA* \\ LUIS ADOLFO MARTÍNEZ-HERRERA**
}

Recibido: 04 de octubre de 2017 Aprobado: 15 de diciembre de 2017

Artículo de Reflexión

\footnotetext{
* Especialista en Psicología Social Comunitaria y Acción Psicosocial. Universidad Católica de Pereira, Pereira, Colombia. E-mail: pamela.santa@ucp.edu.co. D ORCID: 0000-0002-2433-1483. Google Scholar ** Candidato a Doctor en Ciencias Sociales. Docente-investigador Universidad Católica de Pereira, Pereira, Colombia. E-mail: luis.martinez@ucp.edu.co. D ORCID: 0000-0002-0841-932X Google Scholar
} 


\title{
Resumen
}

Objetivo. Reflexionar sobre las dinámicas de reincidencia de las prácticas sociales violentas en la población en proceso de reintegración y su relación con la política nacional de reintegración económica y social, ya que la tasa de reincidencia es significativa. Metodología. Reconociendo los avances y retrocesos se realizó una revisión documental de la normativa vigente y de algunas investigaciones realizadas sobre la reincidencia de la población en proceso de reintegración. Posteriormente se analizaron los factores que explican la reincidencia con un corpus de historias de vida de participantes de la Agencia Colombiana para la Reintegración. Resultados. Se encontró poca información estadística oficial, así como los factores culturales y sociales pueden ser determinantes para la reincidencia más que el factor económico. Conclusión. Es necesario orientar el análisis de la política nacional de reintegración económica y social en las prácticas sociales de la población reintegrada para construir estrategias de larga duración que permitan disminuir la reincidencia.

Palabras clave: práctica social, reincidencia, proceso de reintegración, desmovilizados, grupos armados ilegales.

\section{SOCIAL PRACTICES AND THE RECIVIDISM OF PEOPLE IN THE PROCESS OF REINTEGRATION, IN THE FRAMEWORK OF THE NATIONAL ECONOMIC AND SOCIAL REINTEGRATION POLICY}

\begin{abstract}
Objective. To reflect on the dynamics of recidivism of violent social practices in the population in the process of reintegration and its relationship with the national policy of economic and social reintegration, since the rate of recidivism is significant. Methodology. Recognizing the advances and setbacks, a documentary review of the current regulations and of some investigations carried out on the recidivism of the population in the reintegration process was carried out. Subsequently, the factors that explain the recidivism with a corpus of life stories of participants of the Colombian Agency for Reintegration were analyzed. Results. Little official statistical information was found, as well as it was determined that cultural and social factors may be determinant for recidivism rather than the economic factor. Conclusion. It is necessary to guide the analysis of the national policy of economic and social reintegration in the social practices of the reintegrated population in order to construct long-term strategies that allow the reduction of recidivism.
\end{abstract}

Key words: social practice, recidivism, reintegration process, demobilized, illegal armed groups. 


\section{Introducción}

— 1 proceso de reintegración en Colombia ha tenido sus transformaciones a lo largo del tiempo, en relación con los acuerdos que se han realizado con los grupos armados ilegales para su respectiva reincorporación a la vida civil. Durante este proceso de reintegración se reconocen avances tales como implementarlo a largo plazo, incluir dimensiones en la ruta de la reintegración relacionadas con procesos psicosociales (personal, ciudadano, familiar) y tener experiencias significativas con la población en algunos aspectos. Sin embargo, y considerando que es una política para la reincorporación a la vida civil de las personas que pertenecieron a un grupo armado ilegal ${ }^{1}$, aún se encuentra en proceso de fortalecimiento; máxime, cuando se continúan realizando acuerdos o negociaciones para que los grupos armados ilegales se desmovilicen y continúen sus reivindicaciones por medios legales.

En vista de que el proceso de reintegración es complejo por las condiciones sociales, la polarización social en la que se suscitan los acuerdos con los grupos armados ilegales, la poca participación e inclusión de la sociedad civil en dichos procesos, entre otras, es relevante reflexionar sobre la reincidencia de personas que ingresan al proceso de reintegración. Existen distintos perfiles de reincidentes: los desmovilizados que no ingresan al proceso de reintegración (que según cifras de la Agencia Colombiana para la Reintegración - ACR-, en su informe de 2016, representan el $15 \%$ ), de lo cual no existe mayor información; aquellos que estando en el proceso de reintegración se judicializan por alguna falta o delito y aquellos que han finalizado el proceso de reintegración y han reincidido en algún delito. Sin embargo la cifra oficial acerca de los reincidentes tiende a aumentar, si se considera que no todos los delitos cometidos a partir de la desmovilización llegan a comprobarse. Por esta razón, desde la institucionalidad, se reconoce que son la cuarta parte de la población atendida entre 2003 y 2016 en el marco de la política nacional de reintegración económica y social.

Es importante reflexionar sobre las dinámicas de reincidencia más allá de calificar la pertinencia o calidad de la oferta pública ofrecida en el proceso, a partir de la perspectiva de las prácticas sociales de los agentes que estando en un proceso de reintegración reinciden en prácticas violentas

\footnotetext{
1 "Se entiende por grupo armado organizado al margen de la ley aquel grupo de guerrilla o de autodefensas, o una parte significativa e integral de los mismos como bloques, frentes $u$ otras modalidades de esas mismas organizaciones que, bajo la dirección de un mando responsable, ejerza sobre una parte del territorio un control tal que le permita realizar operaciones militares sostenidas y concertadas" (ACR, 2016, p. 24).
} 
e ilegales sin que ello implique necesariamente el retorno al grupo armado ilegal al que perteneció sino a otras actividades ilegales y violentas. De esta manera la presente reflexión tiene la intención de relacionar las dinámicas de reincidencia, a través de la investigación con historias de vida realizada por Salazar $(2016)^{2}$ debido a que sus entrevistas tienen elementos importantes que le aportan a la reflexión, al indagar por el habitus en esta población.

Este asunto es de interés para la psicología social comunitaria, más aún para la Especialización en Psicología Social Comunitaria y Acción Psicosocial de la Universidad Católica de Pereira; la cual busca aportar desde la academia a la construcción de paz y al proceso de posacuerdo con las FARC-EP. Se analiza la reincidencia en el proceso de reintegración que ha venido realizando la $A C R$, desde las prácticas sociales de los sujetos de esta política nacional, con el propósito de aportar elementos para considerar futuras acciones psicosociales que posibiliten su fortalecimiento.

\section{Situación actual de la reincidencia desde las cifras}

Existe un interés de la academia, las organizaciones sociales y la institucionalidad por interpretar los procesos concernientes a la desmovilización y a la reintegración en el marco de la construcción de paz. Sin embargo no todas las investigaciones ni las estadísticas se centran en la reincidencia, sino en diferentes aspectos que también aportan a la reflexión acerca del proceso de reintegración social y económica de los desmovilizados en el país. Por ello el interés de este análisis es contribuir a la reflexión sobre la reincidencia desde las prácticas sociales, aspecto importante para indicar algunos de los aciertos y desaciertos de la política nacional de reintegración.

Para tal motivo es fundamental mencionar las cifras que se identificaron en los informes realizados por la ACR, la cual tiene a su cargo a nivel nacional este proceso: el porcentaje de personas que permanecen en la legalidad es aproximadamente del 76 \%, entre 2003 y 2017, de acuerdo al último informe de logros (ACR, 2017). Lo anterior como resultado de un estudio adelantado por la Fundación Ideas para la Paz y la Universidad de Columbia (2014), logrando establecer dicha cifra de legalidad y una tasa de reincidencia cercana al $24 \%$ de la población atendida; es decir que una

\footnotetext{
${ }^{2}$ Proyecto de investigación desarrollado por Jean Paul Salazar para obtener el título de psicólogo de la Universidad Católica de Pereira. Proyecto asesorado por el docente-investigador, Luis Adolfo Martínez Herrera. Ambos investigadores accedieron a entregar las entrevistas realizadas para ser analizadas en el presente artículo. Las entrevistas fueron realizadas entre el 1 de junio y el 28 de septiembre de 2016.
} 
cuarta parte de las personas que han ingresado al proceso de reintegración, reinciden. Esto indica que, pese a que la tasa de reincidencia es significativa, en comparación con los procesos de resocialización del INPEC, también es más efectiva debido a que el porcentaje de éxito del INPEC es del $30 \%$ y en cambio el de la ACR es del $70 \%$; sin tener en cuenta que el proceso carcelario lleva 24 años en funcionamiento, mientras que el proceso de reintegración vigente tiene tan solo 13 años.

En el mismo informe se plantea que el $15 \%$ (8784 personas) de los desmovilizados no ingresa al proceso de reintegración (ACR, 2017). Esto indica que es una población que se puede considerar en mayor riesgo de reincidencia, al no realizar un proceso de reincorporación a la vida civil. Por ende, el Estado no le hace seguimiento y no garantiza su proceso. Esta información es relevante como parte del contexto de la problemática, aunque es uno de los perfiles de posibles reincidentes. Así pues, esta reflexión se centrará en aquellas personas que están o han participado del proceso de reintegración debido a que existe poca información acerca de la población que no ingresa a este.
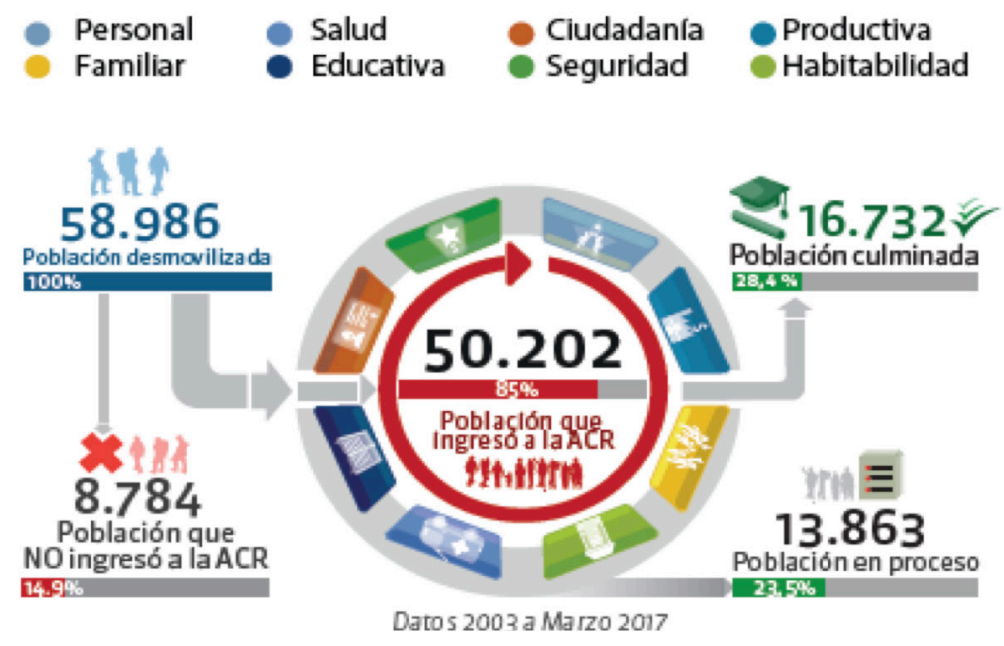

Figura 1. Información sobre la población en proceso de reintegración.

Fuente: ACR (2017).

Otro aspecto importante que devela la ACR es el tipo de grupo armado ilegal al que pertenecían los desmovilizados, al considerar que la mayor parte de la población que ingresó al proceso de reintegración estuvo vinculada a las FARC-EP o a las AUC. Además, la vinculación al grupo armado ilegal permite identificar el posible tipo de desmovilización realizada: colectiva o individual. Por ello es preciso mencionar que los 
desmovilizados de las FARC-EP, que representan el $44 \%$ de la población en proceso de reintegración (ACR, 2017), en su mayoría, desertaron del grupo armado ilegal; sin embargo hay que tener en cuenta que antes de 2016 no existía ningún acuerdo de paz, por lo que la desmovilización se efectuaba de forma individual.

Los desmovilizados de las AUC, a diferencia del proceso con las FARC, que representan el 45,9\% de la población en proceso de reintegración (ACR, 2017), en su mayoría, se desmovilizaron colectivamente debido a las negociaciones consolidadas en la Ley de Justicia y Paz. Esta norma les otorgó el derecho a reincorporarse a la vida civil. La diferencia en la vinculación con el grupo armado ilegal y el tipo de desmovilización cobrará importancia más adelante para la reincidencia.

Después de relacionar los datos que la institucionalidad muestra acerca del proceso de reintegración, es preciso dar claridad sobre el concepto de reincidencia en este contexto. La reincorporación a la vida civil está reglamentada por la Ley 975 de 2005, en su artículo 3, como:

beneficio consistente en suspender la ejecución de la pena determinada en la respectiva sentencia, reemplazándola por una pena alternativa que se concede por la contribución del beneficiario a la consecución de la paz nacional, la colaboración con la justicia, la reparación a las víctimas y su adecuada resocialización.

En este contexto la reincidencia se refiere a la repetición de prácticas delictivas durante o después de un proceso de reintegración, las cuales no solo implican retornar al grupo armado ilegal al que se perteneció sino a toda práctica ilegal y/o violenta que se realice una vez desmovilizado.

Retomando el estudio realizado por la Fundación Ideas para la Paz (2014) que, a partir de un instrumento aplicado a personas en proceso de reintegración, encontró algunos indicios sobre la reincidencia:

se logró establecer que el $20 \%$ de los desmovilizados están totalmente reintegrados, puesto que no tienen ningún valor positivo en las distintas medidas construidas; que el $42 \%$ se encuentra en un riesgo bajo-medio de reincidencia, dado que tienen algún valor positivo en las medidas de vulnerabilidad al reclutamiento o proclividad a la reincidencia; que el $14 \%$ está en un nivel de riesgo medio-alto, puesto que tiene un valor positivo en la medida de reincidencia directa pero no en la binaria, y que el $24 \%$ son desmovilizados plenamente reincidentes, dado que tienen valores positivos tanto en la medida de reincidencia directa como en la binaria. (p. 7) 
Este análisis no solo indica un porcentaje significativo de la población en proceso de reintegración que presenta una disposición a la reincidencia, sino que afirma que una de las razones identificadas que podría explicarla se relaciona con que "los factores asociados con la experiencia misma de la reintegración son predictores más precisos de la reincidencia directa que los factores previos y los asociados al periodo de pertenencia al Grupo Armado ilegal" (Fundación Ideas para la Paz, 2014, p. 8). En este sentido se le otorga un rol determinante al proceso de reintegración para que la transición a la vida civil de los desmovilizados sea efectiva.

Asimismo, en una de las recomendaciones que propone el estudio, se establece una diferenciación entre el tipo de desmovilización y la reincidencia: al considerarse que tienen una mayor disposición para reincidir aquellos que se desmovilizan colectivamente en relación a los que lo hacen de manera individual (Fundación Ideas para la Paz, 2014). Dicho hallazgo, reconoce que el proceso de reintegración es más efectivo en las desmovilizaciones individuales debido a la desvinculación con el grupo armado ilegal por decisión propia. En cambio, las desmovilizaciones colectivas mantienen el vínculo como grupo y corresponden a negociaciones con el gobierno; lo que en el contexto actual podría significar que los desmovilizados de las AUC tendrían una mayor tendencia a reincidir en sus prácticas ilegales, en comparación con quienes desertaron de las FARC-EP. Este último aspecto, será uno de los retos que el proceso de reintegración tendrá que asumir durante la implementación de los acuerdos de paz dado que la desmovilización se está efectuando colectivamente.

La relación entre la desmovilización colectiva y la reincidencia, en el caso de las AUC, parece que se corrobora en otras investigaciones como la realizada por el Centro Nacional de Memoria Histórica - $\mathrm{CNMH}-$ (2015); en donde se establece la relación entre rearmados y reintegrados después de los acuerdos con la AUC. Esta relación se sustentan con el vínculo que se ha venido encontrando entre desmovilizados de las AUC y las BACRIM para realizar prácticas delictivas. La siguiente tabla indica las capturas que se efectuaron entre 2006 y 2009 contra las BACRIM, contra los desmovilizados por tener delitos pendientes, así como precisa aquellos desmovilizados que han sido capturados por un vínculo directo con estas bandas (es decir, reincidentes). 
Tabla 1. Información de capturas de desmovilizados y BACRIM

\begin{tabular}{|c|l|l|l|l|}
\hline & 2006 & 2007 & 2008 & 2009 \\
\hline Capturas Bacrim & 1014 & 1943 & 2163 & 968 \\
\hline Capturas desmovilizados & 1360 & 1612 & 868 & 344 \\
\hline $\begin{array}{c}\text { Capturas desmovilizados } \\
\text { por Bacrim }\end{array}$ & 244 & 349 & 285 & 148 \\
\hline
\end{tabular}

Fuente: CNMH (2015).

En esta dirección, el segundo informe del Centro Internacional de Toledo para la Paz —CITpax- (2009) corrobora indicando que:

según estimativos de la Policía Nacional, de los más de 3.700 miembros que se estiman tenían las bandas emergentes a nivel nacional en mayo de 2009, sólo un 15\% serían desmovilizados. Entre 2005 y 2009, más de 4.700 desmovilizados han sido capturados por reincidir en el delito, de los cuales 1.060 estaban relacionados con las bandas emergentes. (p. 97)

Incluso las cifras sobre la reintegración permiten conocer las dinámicas de reincidencia a nivel regional, con el porcentaje de la suma de la población que ha perdido los beneficios por dicha causa, así se observa en los siguientes departamentos: Risaralda (30,9\%), Meta (31,6 \%) y Valle del Cauca $(34,2 \%)$, que superan el promedio nacional de reincidencia que es del 30,8\%; por el contrario, Caldas (22,7\%) o Magdalena (21,8 \%) están por debajo del promedio (ACR, 2016). Estos promedios corroboran que la tasa de reincidencia es significativa con tendencia a aumentar, lo que requiere que el proceso de reintegración deba fortalecerse para lograr re-significar efectivamente las prácticas sociales de los desmovilizados en la vida civil.

\section{La reincidencia y las condiciones sociales}

La reincidencia se presenta por diferentes factores, lo que denota su complejidad. Las cifras no abarcan en su totalidad la complejidad, desde la perspectiva psicosocial y relacional, que evidencia los factores que inciden en que una persona en proceso de reintegración llegue a reincidir en prácticas sociales violentas o ilegales ${ }^{3}$. Es por eso que esta reflexión

\footnotetext{
3 “Las prácticas sociales violentas se entienden no sólo como expresiones fácticas de acciones mediadas por la fuerza — sea esta física o simbólica—sino —en especial—como propensiones para la acción, como disposiciones aprehendidas en unos marcos institucionales los cuales encuentran en el llamado sub-campo de la economía ilegal el nodo relacional que vincula las dimensiones legales, grises e ilegales de la sociedad Risaraldense y que socializan lógicas de acción mediadas por el uso de múltiples formas de violencia" (Martínez, 2017, p. 22).
} 
pretende realizar un acercamiento a esta perspectiva. Para ello es necesario relacionar la reincidencia con las condiciones sociales como una línea para entender dicha situación, al considerar que algunas investigaciones ya han identificado factores que pueden influir en los desmovilizados para su reincidencia.

Para analizar el proceso de reintegración, y con el ejemplo de la ciudad de Pereira, se utilizó un corpus de historias de vida de desmovilizados de las AUC y las FARC-EP (Alvaro, Mario, Luisa y Jorge) recolectadas por Salazar (2016) en el marco de su trabajo de grado y del proceso investigativo del semillero de investigación "Figuraciones de la violencia en el Eje Cafetero" adscrito al grupo de investigación "Comunicación y conflicto" de la Universidad Católica de Pereira. Los datos fueron obtenidos a través de entrevistas para la construcción de historias de vida por una matriz de preguntas orientadoras, las cuales se realizaron con la autorización de la ACR en el mes de noviembre de 2016. Los informantes son personas que se encontraban en proceso de reintegración en la ACR seleccionados de manera aleatoria, lo que permitió identificar una condición social común entre ellos: la presencia de grupos armados ilegales en su territorio; hay que tener en cuenta que cada informante es de un lugar de origen diferente y que ellos ocuparon diversos roles en la estructura de los grupos armados ilegales.

El proceso de análisis se dividió en varias fases: en la primera se realizó una revisión documental en relación a la normativa vigente en torno a la política nacional de reintegración económica y social, los informes estadísticos de la ACR y las diferentes investigaciones que se han realizado sobre la reincidencia en desmovilizados de grupos armados ilegales; en un segundo momento se relacionan los resultados y análisis de las investigaciones revisadas con las categorías de campo, habitus y prácticas sociales, desde la perspectiva relacional de Pierre Bourdieu; en el tercer momento se contrasta el análisis relacional realizado con el discurso de los informantes en sus historias de vida, con el fin de reflexionar en torno a los factores que pueden influir en que una persona en proceso de reintegración reincida en la ilegalidad.

Fue así como se encontró en las historias de vida, que los grupos armados ilegales realizaban estrategias que permitían tener un acercamiento con la comunidad para influir en la toma de decisiones; tal como lo menciona Álvaro": "en la zona en la que vivía era común la presencia de guerrillas, las cuales establecían normas e indicaciones a las Juntas de acción comunal y

\footnotetext{
${ }^{4}$ Álvaro es la primera historia de vida referida por Salazar (2016); es un desmovilizado de las FARC-EP, quien estuvo 14 años y tuvo un rol de mando en el grupo armado.
} 
realizaban reuniones en diferentes escenarios comunitarios"; se evidencia que el grupo armado ilegal, al ser un agente familiar para Álvaro, facilitó su ingreso gracias a las percepciones que él mismo había construido de ese grupo a través de sus prácticas con la comunidad. Situación que puede reproducirse con otras características en su contexto y con otro tipo de grupos armados que operen en el mismo.

Desde este punto de vista es pertinente analizar dichos factores de manera relacional, ya que para Bourdieu (1988) “existen en el mundo social [...] estructuras objetivas, independiente de la conciencia y de la voluntad de los agentes, que son capaces de orientar o de coaccionar sus prácticas o sus representaciones" (p. 127). Por tanto, es preciso reconocer que existen condiciones sociales y económicas que pueden estar relacionadas con la reincidencia de la población en proceso de reintegración; en vista de ello hay que tener en cuenta que la reintegración social y económica a la vida civil es compleja por su historia, sus prácticas, sus percepciones, sus representaciones, las de los demás agentes en el espacio social y en la trayectoria entre el grupo armado ilegal y la vida civil.

Algunas investigaciones resaltan como el factor económico se relaciona con la reincidencia en los desmovilizados (CNMH, 2015). En este sentido se centra la investigación de Garay (2016), quien afirma que "el crimen aumentará cuando criminales potenciales (desmovilizados) presenten dificultades en encontrar trabajos legales, debido, quizás, a una tasa desempleo alta o porque las personas abandonan la escuela con pocas habilidades" (p. 22); denotando como la falta de garantías económicas favorece la reincidencia, aun más en un contexto donde no está garantizada esa estabilidad económica en la vida civil.

$\mathrm{Al}$ respecto es importante mencionar que para Bourdieu el capital es la razón de ser del campo (como se citó en Gutiérrez, 2005) y que dicha distribución, que es desigual, configura las posiciones de los agentes que están en el juego luchando por un bien preciado que es necesario obtener para ser parte del mismo. Esto se puede resumir en palabras de Bourdieu (1976): "para que funcione un campo, es necesario que haya algo en juego [capital] y gente dispuesta a jugar [agentes y/o instituciones]" (p. 136). La categoría de capital nos permitirá entender las tensiones entre los agentes que están en el campo dado el volumen de capital que cada agente posee. Por esta razón no solo se debe reflexionar si el proceso de reintegración proporciona las necesidades básicas, sino en qué condiciones; y si al concluir el proceso se garantiza que los reintegrados vivan en la legalidad (considerando las altas tasas de desempleo y el empleo informal a nivel nacional, solo como uno de los aspectos a tener en cuenta). 
Para continuar es necesario retomar el vínculo mencionado entre los desmovilizados reincidentes y las BACRIM no solo en torno a las cifras que presentan el CNMH y el CITpax, sino en el sentido indicado por Garay (2016): ya que los grupos armados ilegales y las BACRIM tienen un modus operandi con algunas similitudes entre sí, que podría facilitar el ingreso a estas últimas como sucedió anteriormente. Así pues, tiene relevancia lo que Garay (2016) menciona al respecto:

la ubicación de los excombatientes desmovilizados, en su mayoría, se encuentra en las localidades donde hay mayor presencia de crimen; lo que unido a bajos niveles de educación, pocas habilidades o contactos sociales (CONPES 3554, 2008) puede generar una influencia notable en la decisión tomada por las PPR sobre actividades delictivas. (p. 23)

En relación al modus operandi de los grupos armados se observa algunas características similares tales como la presencia permanente en el territorio, el interés por influir en las decisiones de la comunidad, el interés por consolidar el capital económico y simbólico que contribuya a alcanzar sus objetivos, el establecimiento de normas y prácticas sociales utilizando la fuerza entre otras. Por tanto, no es una coincidencia que parte de los reincidentes ingresen a las BACRIM por tener prácticas sociales que le son familiares a los desmovilizados y aunadas a unas condiciones sociales que no garantizan el bienestar integral que ellos esperan.

\section{La reincidencia y el habitus como generador de prácticas}

Desde una perspectiva relacional, así como se resalta las condiciones sociales en la reincidencia, son importantes las percepciones y representaciones que el agente construye de su realidad social para realizar determinadas prácticas. Por esta razón es pertinente retomar a Bourdieu (1980), quien señala cómo "los condicionamientos asociados a una clase particular de condiciones de existencia producen habitus [...] como principios generadores y organizadores de prácticas y representaciones que pueden estar objetivamente adaptadas a su fin sin suponer la búsqueda consciente de fines" (p. 92); haciendo mención de cómo las dinámicas del campo social donde han vivido los agentes posibilitan construir pensamientos, percepciones y representaciones sociales que pueden facilitar y justificar la reincidencia.

Para ello es preciso resaltar la relación entre desmovilizados y Estado como uno de los aspectos para evidenciar el habitus, develando la percepción que tienen los propios participantes del proceso de reintegración frente a 
lo que brinda la institucionalidad. Ugarriza y Mesías (2009) señalan como las expectativas de los desmovilizados no corresponden con los beneficios recibidos o con lo prometido al ingresar al proceso, ya que la lucha del grupo armado ilegal fue en contra de un Estado negligente y ausente. Así pues,

el binomio engaño/desconfianza se presenta entonces como un problema estructural que afecta transversalmente el proceso de reintegración. Identificar las distancias que existen entre la política de reintegración y las demandas de los desmovilizados permite dimensionar los problemas generales del actual proceso. (Ugarriza y Mesías, 2009, p. 10)

Participar en el proceso de reintegración no significa que las condiciones sociales hayan mejorado, ni que las causas que en un inicio motivaron la conformación de los grupos armados ilegales hayan sido resueltas; aquel que se desmoviliza abandona la lucha armada, cambia de posición, pero eso no implica que confíe en el Estado ni que sus expectativas frente a la reintegración sean realizadas. La dificultad no se centra en la desconfianza hacia el Estado, a pesar de que dicho sentimiento también es compartido por miles de ciudadanos que no han abandonado la vida civil, aunque sí puede influir en la medida en que la vida civil no le permita gozar de algún tipo de bienestar y encuentre en la reincidencia a la ilegalidad - de antemano con una experiencia previa - su bienestar.

Aquí cobra relevancia la noción de trayectoria de Bourdieu puesto que permite reconocer que las "semejanzas entre los sistemas de disposiciones de los individuos comparten similares condiciones objetivas de vida —condiciones de clase-" (Gutiérrez, 2005, p. 80); sin embargo los "sistemas de disposiciones [habitus] no son necesariamente iguales, sino que cada uno de ellos se diferencia de los otros por la singularidad de la trayectoria social" (Gutiérrez, 2005, p. 80). Desde esta perspectiva es posible entender el por qué una parte de la población en proceso de reintegración reincide; además, justifica la importancia de analizarla relacionalmente a partir de las prácticas sociales.

Pese a la semejanza en los sistemas de disposiciones es relevante reconocer la trayectoria modal del grupo armado ilegal para identificar las relaciones de poder, la dinámica del campo social, su habitus y los capitales en juego. Por tanto, para realizar el proceso de reintegración, es fundamental analizar la singularidad de la trayectoria social. Por ejemplo, es diferente la persona que ha pertenecido a un grupo armado ilegal 
durante 14 años (Álvaro) a la que ha permaneció 5 o 7 años $\left(\right.$ Mario $^{5}$, Luisa ${ }^{6}$ y Jorge $\mathrm{e}^{7}$; los que fueron reclutados forzosamente (Luisa) respecto a los que decidieron ingresar al grupo voluntariamente (Álvaro, Mario y Jorge); quien fue capturado (Jorge) al que desertó del grupo armado (Álvaro, Luisa y Mario); quienes se desmovilizaron colectivamente (AUC) a quienes se desmovilizaron individualmente (FARC-EP); quien tuvo un rol de mando (Álvaro) a quien fue soldado raso (Mario, Luisa y Jorge).

Por otra parte se muestra como la justicia social, el servicio a la comunidad y el rechazo a la corrupción se convierten en el interés común entre el grupo armado y los desmovilizados antes de ingresar a él; siendo estas algunas de las motivaciones que favorecen su vinculación a la lucha armada como una opción para alcanzarlas. Por ejemplo, Álvaro manifiesta "mira que habían normas, cuestiones de que si ayudaban a la comunidad", "de esta forma se llegaban a hacer cambios en las regiones, hacer proyectos"; mientras que para Jorge "el tema de la justicia social, porque yo veía que era una gente que ayudaba muchísimo, eso me gustaba mucho y uno veía el progreso de la región", "una de las cosas que más me llamó la atención de las FARC fue lo fuerte que era [...] con el tema de la corrupción"; dichas afirmaciones reflejan como su habitus se identificaba con el habitus del grupo armado ilegal.

Estas razones esgrimidas como principios de acción justifican las prácticas sociales violentas e ilegales, ya que el habitus es generador de las prácticas que el agente realiza. De esta manera, al percibir que la vida civil no ofrece lo que buscaba, puede reincidir en las bandas criminales cercanas a su campo social; esto, motivado por la falta de estabilidad económica, por la falta de seguridad física, por la falta de participación política para aquellos que persiguen la justicia social entre otras (Nussio, 2009). Nussio (2009) y Ocampo (2014) afirman que la reincidencia desde múltiples factores —como los ya mencionados- puede incidir en dicha práctica, específicamente en el caso de las AUC en el marco de la Ley de Justicia y Paz.

Nussio (2009), además, devela un aspecto importante en relación al capital simbólico que los desmovilizados construyen en su permanencia en el grupo armado: "con el desarme y la desmovilización pierden este estatus adquirido como combatientes. La pérdida de prestigio social puede ser un incentivo para afiliarse otra vez a grupos armados" (p. 221);

\footnotetext{
${ }^{5}$ Mario es la segunda historia de vida referida por Salazar (2016); es un desmovilizado de las FARC-EP, quien estuvo 5 años en el grupo armado.

${ }^{6}$ Luisa es la tercera historia de vida referida por Salazar (2016); es una desmovilizada de las FARC-EP, quien estuvo 7 años y medio en el grupo armado y manifiesta haber sido reclutada.

${ }^{7}$ Jorge es la cuarta historia de vida referida por Salazar (2016); ingresó al proceso de reintegración al ser capturado, estuvo 5 años en las FARC-EP.
} 
asunto que al interior de la ruta para la reintegración se encuentra en proceso de fortalecimiento de la dimensión ciudadana encaminada a la reintegración comunitaria.

La posición social que cada desmovilizado construye es clave en dicho proceso puesto que las percepciones, representaciones y prácticas que haya tenido facilitarán o impedirán la reintegración social y económica. Nussio (2009) y Ocampo (2014), en sus respectivas investigaciones, ponen como ejemplo el sentido que para ellos tiene poseer un arma: ya que no solo les puede significar poder, dominio y fuerza, sino también protección y supervivencia; es así como al despojarse de dicho objeto pueden sentirse vulnerables y desprotegidos, representando una pérdida de su capital simbólico.

Nussio (2009) también considera como otro de los factores para la reincidencia la falta de aceptación social por parte de agentes e instituciones que se encuentran en el campo social, pues existe una parte de la población que no está de acuerdo con la reintegración social y económica de los desmovilizados, lo que complica la transición del desmovilizado entre ser un actor armado a ser un ciudadano. Por esta razón es necesario reconocer las múltiples tensiones en torno a la reintegración para no solo centrarse en las prácticas sociales de los desmovilizados, sino en las prácticas de la sociedad civil que favorezcan la reincorporación efectiva.

Es importante considerar como para algunos desmovilizados no todo lo vivido durante su permanencia en el grupo armado fue negativo dado a que en dicha experiencia también construyeron relaciones, conocimientos, habilidades, rutinas, costumbres, percepciones, valores y normas que son incorporadas y conservadas en su habitus. Este es el caso de las FARC-EP, que lleva a cabo una formación ideológica permanente donde especialmente los valores y las normas no se cuestionan sino que se obedecen.

Esta situación se reconoce en la entrevista realizada a Jorge: "yo creo que el hecho de haber salido del grupo no significa que tenga que dejar todo allá y olvidar lo bonito que también aprendí en el grupo"; por un lado, Álvaro destaca que algunos principios o valores éticos aprendidos se mantienen como lo es "esa mentalidad de querer ayudar"; por otro, Luisa manifiesta ser solidaria "eso como que se me quedó de allá, a compartir, igual le enseño a mis hijos como a tener ese compañerismo"; mientras que Mario exalta como "la disciplina allá es bastante, el cumplir y respetar a la autoridad, que las personas opinen [...] eso también son cosas positivas que me dejo el grupo".

Lo anterior significa que no todo habitus ni las prácticas que los agentes incorporaron en el grupo armado desaparecen al momento de realizar un proceso de reintegración sino que se pueden conservar, transformar o 
reproducir (Bourdieu, 1980, 1988), aspecto que se debe tener en cuenta en el proceso de reintegración para prevenir o identificar alguna propensión para la reincidencia.

\section{Las prácticas sociales y la reincidencia desde una perspectiva relacional}

La categoría de prácticas sociales de Bourdieu nos permite reflexionar acerca de la reincidencia de manera relacional puesto que ayuda a considerarla pluridimensionalmente; en donde no solo involucra al desmovilizado y a la institucionalidad sino que reconoce la historia de vida de cada sujeto, su habitus, su singularidad en la trayectoria social, así como las relaciones de poder con otros agentes que se encuentran en el campo social.

Para reflexionar desde la perspectiva relacional es necesario comprender que:

las estructuras sociales existen dos veces, que lo social está conformado por relaciones objetivas, pero que también los individuos tienen un conocimiento práctico de esas relaciones - una manera de percibirlas, de evaluarlas, de sentirlas, de vivirlas-, e invierten ese conocimiento práctico en sus actividades ordinarias. (Gutiérrez, 2005, p. 18)

En esta medida las prácticas sociales dan la posibilidad de reconocer en el agente, en este caso en el desmovilizado que reincide en la ilegalidad, sus percepciones, sus apreciaciones y sus representaciones construidas históricamente no solo por la trayectoria individual sino por la trayectoria modal que ha vivido su clase y que a la vez ha incorporado para estar en el espacio social.

El habitus que un desmovilizado ha construido antes, durante y después de pertenecer al grupo armado ilegal, posibilita comprender las motivaciones que tiene una persona para reincidir en sus prácticas sociales violentas después de estar en un proceso de reintegración; y la implicación que tiene desmovilizarse individual o colectivamente, pues es probable que la tendencia a la reincidencia sea más frecuente cuando la desmovilización es colectiva que cuando es individual.

Complementario a lo anterior, las prácticas sociales también se afectan por las tensiones que existen entre los campos y por las estructuras sociales externas que generan que un agente reproduzca su habitus y las prácticas en relación al interés que se encuentra en juego en el espacio social; pues, como ya se menciono, el capital es la razón de ser del campo. En este sentido juega en la reincidencia la relación del desmovilizado con 
la institucionalidad, en donde pondrá en tensión su habitus y el capital que conserva o que modifica en contraste con el que poseía en el grupo armado. Por ello en los antecedentes de los factores de reincidencia se identifican la poca aceptación social y la pérdida de prestigio, esto dependiendo de la posición que ocupaba dentro del grupo armado.

En las relaciones de poder los desmovilizados se enfrentan a una particular tensión. Nussio (2009) menciona que los participantes en el proceso de reintegración se encuentran en "una franja gris de la economía entre lo lícito y lo ilícito" (p. 218); al considerar sus historias de vida, sus prácticas, sus representaciones, sus percepciones construidas durante el tiempo que pertenecieron al grupo armado, bajo el marco de la ilegalidad y la violencia; en contraste con el tránsito a la vida civil que implica otras prácticas, otras representaciones y otras vivencias.

El tránsito complejo entre estas dos experiencias de vida puede generar en el sujeto crisis mientras afronta su nuevo contexto; aunado a un sentimiento de inconformidad con el proceso de reintegración, con los beneficios económicos y con la no aceptación social, que lo lleven a retornar a la reincidencia. Por ello es importante reconocer las condiciones sociales e internalizadas que puedan identificar factores de riesgo singular para la reincidencia en los desmovilizados.

Porconsiguiente, las prácticas sociales violentas que realizaban durante su pertenencia al grupo armado correspondían al habitus incorporado dado que para dichas acciones tenían una justificación coherente con su justicia social; para Álvaro: “cuando estás adentro lo ves y lo haces de una manera que de pronto es una estrategia [...] para sacudir y presionar al Estado" y "que un soldado fue secuestrado, nunca es secuestrado [...] [son] prisioneros de guerra"; es decir que desde que exista una motivación que corresponda al habitus del grupo armado este es aceptado y realizado a través de sus prácticas sociales: "uno cree que lo que está haciendo está bien hecho" (Lucia).

Incluso en el caso de Jorge, quien fue capturado y se está reintegrando, se evidencia la diferencia entre la percepción al estar dentro o fuera del grupo armado: "en la televisión se ve una FARC muy diferente a la que realmente convivía con nosotros"; además, complementa, "uno creía que por ejemplo cuando ellos ejecutaban a alguien, lo ejecutaba justamente pues porque era vicioso, era ladrón"; en esta situación se puede demostrar el sistema de valores incorporado, en el que consideraba que una persona que fuera viciosa o ladrona merecía ser ejecutada.

Las prácticas sociales corresponden a la relación entre las estructuras sociales externas (campo) y las estructuras sociales incorporadas (habitus). De esta manera, si no es posible transformar estas estructuras en los 
desmovilizados, es posible que se generen dinámicas de reincidencia a la ilegalidad y a la violencia a través de bandas criminales por medio de los factores expuestos que pueden favorecer la reincidencia durante o después del proceso de reintegración.

\section{Conclusiones}

Durante el proceso de reintegración se torna necesario analizar las prácticas sociales de los desmovilizados para el fortalecimiento del proceso de reincorporación 'real' a la vida civil, construyendo estrategias y acciones psicosociales que permitan identificar alertas tempranas para prevenir dinámicas de reincidencia en los desmovilizados. Este análisis debe hacerse de manera relacional y pluridimensional con el propósito de problematizar el fenómeno, identificar los aspectos relevantes que contribuyan a la reincidencia puesto que no es posible evidenciar las prácticas sociales violentas y/o ilegales de los agentes sin reconocer las estructuras sociales externas e internalizadas que lo condicionan.

Para ello es necesario que la política nacional no solo sea monitoreada y centrada en la cifras, sino en el proceso e impacto singular de cada participante de la reintegración; posibilitando dentro del acompañamiento psicosocial la identificación de las percepciones, apreciaciones, valores y representaciones sociales con el propósito de evaluar el impacto de las acciones realizadas en el habitus y prácticas sociales de los participantes. Por consiguiente, es necesario fomentar la investigación social como parte de los procesos internos en la ACR y continuar estimulando las investigaciones desarrolladas por la academia y las organizaciones sociales para el fortalecimiento de esta política.

Es importante que para el cumplimiento de los objetivos de la política nacional, encaminados a la reintegración social y económica de los desmovilizados, se considere el análisis de las prácticas sociales de los participantes desde sus historias de vida para la puesta en marcha de las dimensiones de la ruta de la reintegración; ya que las dinámicas de reincidencia demuestran que no es suficiente con los beneficios de tipo económico, educativos o laborales para prevenir la reincidencia, sino que se requiere de un proceso psicosocial enfocado en la transformación de las prácticas sociales que propicie la vida civil a través de la legalidad y que garantice sus derechos fundamentales.

Otras de las conclusiones que se generan de esta reflexión es la necesidad de fortalecer las acciones psicosociales con la sociedad civil y la comunidad en general, en especial en el entorno más cercano del desmovilizado, con el propósito de promover valores y prácticas sociales 
que fomenten la aceptación social frente a la población reintegrada que le apuesta a la reincorporación efectiva a la vida civil; dado que la no aceptación social se puede convertir en un obstáculo para dicha población. Así, la pertinencia de la política nacional se verá reflejada sí logra incidir en la percepción y las representaciones sociales que se han construido acerca del proceso de reintegración en la sociedad civil y en los propios participantes; pues, de lo contrario, perdería el sentido de su implementación.

Finalmente se requiere que desde la institucionalidad y las organizaciones sociales se realice un monitoreo constante de la reincidencia, esto como una de las categorías centrales para la evaluación de la política nacional; en donde las cifras sean más precisas y específicas frente a las dinámicas de reincidencia para tener más claridad sobre esta problemática. Para este propósito es fundamental que la articulación con las instituciones de justicia sean efectivas para no propiciar escenarios permisivos frente a la reincidencia de las prácticas sociales violentas o ilegales por parte de los reintegrados que puedan favorecer la ilegalidad. Para lograr el fortalecimiento del proceso de reintegración y la no reincidencia es fundamental la participación y la incidencia de los actores sociales, incluidos los mismos participantes, en la construcción de paz.

\section{Referencias bibliográficas}

ACR. (2016). Banco terminológico. Recuperado de https://www.reintegracion.gov.co/es/ agencia/Documentos\%20de\%20Gestin\%20Documental/BANCO_TERMINOLOGICO_ ACR.pdf.

ACR. (2017). La reintegración en Colombia. Recuperado de http://www.reintegracion.gov.co/ es/la-reintegracion/Paginas/cifras.aspx.

Bourdieu, P. (1976). Sociología y cultura. Algunas propiedades de los campos. Ciudad de México, México: Grijalbo.

Bourdieu, P. (1980). Sentido práctico. Estructura, habitus y prácticas. Madrid, España: Taurus Humanidades.

Bourdieu, P. (1988). Cosas dichas. Espacio social y poder simbólico. Buenos Aires, Argentina: Gedisa.

CNMH. (2015). Rearmados y reintegrados. Panorama posacuerdos con las AUC. Bogotá, Colombia: CNMH.

CNMH. (2015). Desmovilización y reintegración paramilitar. Panorama posacuerdos con las AUC. Bogotá, Colombia: CNMH.

CITpax. (2009). Segundo informe. Recuperado de http://www.toledopax.org/sites/default/ files/CITpax_Segundo_Informe_Observatorio_DDR_Ley_Justicia_y_Paz_Colombia_ noviembre_2009.pdf.

Fundación Ideas para la Paz. (2014). Retorno a la legalidad o reincidencia de excombatientes en Colombia: dimensiones del fenómeno y factores de riesgo. Recuperado de http://cdn.ideaspaz.org/media/website/document/53c448d20a31f.pdf. 
Garay, D. (2016). Probabilidad de reincidencia criminal en personas vinculadas al proceso de reintegración social en Bogotá. Bogotá, Colombia: Pontificia Universidad Javeriana.

Gutiérrez, A. (2005). Las prácticas sociales: una introducción a Pierre Bourdieu. Buenos Aires, Argentina: Ferreyra Editor.

Martínez, L. (2017). Contrabando, narcomenudeo y explotación sexual en Pereira, Colombia. Revista Mexicana de Sociología, 79 (3), 459-486.

Nussio, E. (2009). ¿Reincidir o no? Conceptos de la literatura internacional aplicados al caso de desarme, desmovilización y reintegración de las Autodefensas Unidas de Colombia. Pensamiento Jurídico, 26, 213-236.

Ocampo, M. (2014). Criminalidad, grupos armados y reinserción: perfiles y motivaciones. Ciencias Sociales y Educación, 3 (5), 17-57.

Salazar, J. (2016). Habitus ciudadano: una interpretación de las personas desmovilizadas en la ciudad de Pereira (tesis de pregrado). Universidad Católica de Pereira, Pereira, Colombia.

Ugarriza, J. y Mesías, L. (2009). Dilemas de la reintegración de excombatientes en Bogotá. En VIII Seminario de Investigación Urbano-Regional. Universidad Nacional, Bogotá, Colombia. 